\title{
Capnocytophaga ochracea infection: two cases and a review of the published work
}

\author{
PM HAWKEY, H MALNICK, ${ }^{*}$ SAM GLOVER, $\dagger$ N COOK, JA WATTS
}

From the Department of Microbiology, Bristol Royal Infirmary, Bristol BS2 $8 \mathrm{HW}$, the *National Collection of Type Cultures, Central Public Health Laboratory, Colindale, London NW9 5HT, and the †Department of Microbiology, Royal Hallamshire Hospital, Sheffield S10 2JF

SUMMARY Bacteria of the genus Capnocytophaga are recently recognised pathogens which may cause oral disease and subsequent septicaemia in the immunocompromised host. We present two cases of infection caused by Capnocytophaga ochracea; a soft tissue infection in an immunologically normal patient and an episode of septicaemia in a child with leukaemia. The microbiology, pathogenicity, and antimicrobial susceptibility of the genus capnocytophaga are reviewed.

Capnocytophaga ochracea (formerly Bacteroides ochracea) is a non-sporing, microaerophilic, Gram negative bacillus that may produce a yellow pigment on solid media. It is part of the normal oral flora of humans and causes periodontitis. ${ }^{12}$ Recently, there has been considerable interest in this organism as an opportunistic pathogen in immunosuppressed patients, particularly in the USA.$^{3-5}$ We report here two cases of infection, one in a non-compromised host, and review the published work.

\section{Case reports}

CASE 1

A 50 year old women presented with a 10 day history of a painful submandibular swelling anterior to the right sternomastoid. Pain in the region of the swelling had prevented her eating and she had taken only fluids for the preceding four days. She gave a history of a similar episode occurring seven years previously. Aspiration of the mass at the time had yielded pus, a Gram film of which showed many polymorphonuclear cells and no organisms; it was sterile on culture (incubated for $48 \mathrm{~h}$ only). The infection was treated with oral ampicillin and the acute inflammation resolved, leaving a small subcutaneous nodule. Five months before presentation on this occasion she had experienced an episode of pain and swelling in the same region, and this had responded to a course of penicillin $\mathrm{V}$. She gave no history of tuberculosis, diabetes, or any other disease affecting her immunity to infection.

Accepted for publication 13 June 1984
On examination she was apyrexial and had a $5 \mathrm{~cm}$ $\times 4 \mathrm{~cm}$ swelling below the angle of the right mandible and anterior to the sternomastoid. The mass was smooth, fluctuant, and fixed deeply, and there was overlying cellulitis. Both parotid and submandibular ducts were normal and no calculi were felt in the floor of the mouth; radiological examination of the region confirmed the absence of calculi.

Surgical drainage of the mass showed a large cavity containing pus; the cavity was curretted and packed with Eusol and paraffin soaked gauze. A course of cephradine and penicillin $V$ was given and the patient made an uneventful recovery. A Gram stained film of the pus showed many polymorphonuclear cells and no visible organisms, and a Ziehl-Neelsen stain was negative for acid fast bacilli. Bacterial culture at $48 \mathrm{~h}$ was negative, but prolonged culture (five days) yielded a heavy growth of a slender, tapering, Gram negative bacillus, which formed dirty yellow, flat, "pitting" colonies with spreading finger like projections. The isolate was oxidase and catalase negative; it failed to grow on MacConkey agar but grew well on media containing blood incubated in a $\mathrm{CO}_{2}$ enriched atmosphere. Disc sensitivity testing showed it to be sensitive to benzylpenicillin, erythromycin, ampicillin, and clindamycin but resistant to cephradine and metronidazole. It was subsequently identified at the National Collection of Type Cultures as Capnocytophaga ochracea, (strain no A349/83); the biochemical reactions are given in the Table.

This patient had severe local soft tissue sepsis caused by $C$ ochracea, which responded to surgical drainage and an appropriate antibiotic. The earlier episodes of infection at the same site could well have 1066 
Biochemical characteristics of Capnocytophaga ochracae A319/83 and A349/83 isolated from patients described in this paper compared with results obtained by Socransky et al ${ }^{2}$ (68 strains examined)

\begin{tabular}{|c|c|c|c|c|c|}
\hline & $A 319 / 83$ & $A 349 / 83$ & C ochracea & C sputigena & C gingivalis \\
\hline \multicolumn{6}{|l|}{ Acid from: } \\
\hline Dextrin & + & + & NT & NT & NT \\
\hline Fructose & + & + & 89 & 50 & 12 \\
\hline Galactose & + & + & 83 & 0 & 0 \\
\hline Glucose & + & + & 100 & 100 & 100 \\
\hline Glycerol & - & - & NT & NT & NT \\
\hline Lactose & + & + & 92 & 40 & 8 \\
\hline Maltose & + & + & 100 & 100 & 100 \\
\hline Mannitol & - & - & 0 & 0 & 0 \\
\hline Mannose & + & + & 100 & 100 & 100 \\
\hline Raffinose & + & + & 70 & 17 & 24 \\
\hline Salicin & - & - & 11 & 0 & 0 \\
\hline Sorbitol & - & - & 0 & 0 & 0 \\
\hline Starch & + & + & 96 & 60 & 9 \\
\hline Sucrose & + & + & 100 & 100 & 100 \\
\hline Trehalose & - & - & 9 & 0 & 0 \\
\hline \multicolumn{6}{|l|}{ Hydrolysis of: } \\
\hline Aesculin & + & + & 96 & 83 & 75 \\
\hline Starch & + & + & 77 & 0 & 0 \\
\hline Urea & - & - & 14 & 0 & 12 \\
\hline Catalase & - & - & 0 & 0 & 0 \\
\hline Oxidase & - & - & 0 & 0 & 0 \\
\hline Arginine dihydrolase & - & - & 0 & 0 & 0 \\
\hline Lysine decarboxylase & - & - & 0 & 0 & 0 \\
\hline Ornithine decarboxylase & - & - & 0 & 0 & 0 \\
\hline Nitrate reduction & + & - & 8 & 83 & 4 \\
\hline Nitrite reduction & + & + & 57 & 40 & 60 \\
\hline
\end{tabular}

The methods of Cowan and Steel ${ }^{12}$ were used with the following modifications: sodium nitrate for nitrate reduction and $0 \cdot 4 \%$ starch for starch hydrolysis. Phenol red broth base (Difco) was used for acid production from sugars.

$\mathrm{NT}=$ not tested.

been due to the same organism; Gram film and culture results (at $48 \mathrm{~h}$ ) were identical to those found later. On both occasions the symptoms had settled on a course of an appropriate antibiotic. This case illustrates the need to incubate cultures for five days if infections caused by Capnocytophaga spp are not to be missed. Also, the slender Gram negative rods may not be seen in films with large numbers of polymorphonuclear leucocytes.

CASE 2

An 11 year old boy, with previously diagnosed acute lymphoblastic leukaemia, presented with a two day history of diarrhoea and fever. He was receiving intrathecal methotrexate injections for a central nervous system relapse of his disease. On examination he was feverish, looked ill, and had some abdominal tenderness. Blood cultures were taken, and the patient was given ceftazidime. After an initial improvement his condition deteriorated and amikacin was added. After several days he gradually became apyrexial and was discharged. On subculture after four days' incubation a Gram negative bacillus was isolated from the blood cultures. Disc sensitivity testing showed it to be sensitive to penicillin, ampicillin, chloramphenicol, azlocillin, amikacin, and ceftazidime but resistant to gentamicin and tobramycin. The organism was later identified at the National Collection of Type Cultures as $C$ ochracea (strain no A319/83).

This child had an episode of septicaemia caused by $C$ ochracea, which responded to treatment with a combination of ceftazidime and amikacin. The case shows the importance of searching for and identifying unusual bacteria isolated from immunocompromised patients.

\section{Discussion and review}

\section{TAXOMONY AND IDENTIFICATION}

Capnocytophaga ochracea belongs to the class Flexibacteriae, one of three groups of bacteria characterised by their gliding motility on solid surfaces. The genus Capnocytophaga resides within the family Cytophagaceae, which includes species that are common bacteria in soil and water environments. The taxonomy of the gliding bacteria has been comprehensively reviewed, ${ }^{6}$ as have the characteristics of the genus Capnocytophaga. ${ }^{7}$ Until recently, the taxonomy of these organisms was rather confused. Two independent reports in 1979, however, established that Capnocytophaga, Bacteroides ochraceus and CDC bio-group DF-1 were synonymous. ${ }^{89}$

On the basis of biochemical, morphological, and DNA base homology studies ${ }^{10}$ Leadbetter and his colleagues proposed that the genus should contain three species: $C$ ochracea, $C$ gingivalis, and $C$ 
sputigena. "The biochemical criteria for the separation of these three species were also reported by Socransky et $a l,{ }^{2}$ and are compared in the Table with the findings in the cases reported here. The general characteristics of the genus are that they are slender, fusiform, Gram negative bacilli, which grow in an anaerobic or $\mathrm{CO}_{2}$ enriched atmosphere but not in air. They are slow growing at $37^{\circ} \mathrm{C}$, oxidase and catalase negative, and do not grow on MacConkey's agar. They produce a yellow pigment on solid media and may exhibit gliding motility, often best shown on $3 \%$ trypticase soya agar supplemented with $5 \%$ sheep blood. ${ }^{11}$ Sugars are metabolised fermentatively even in the presence of oxygen. The useful biochemical tests to identify the genus Capnocytophaga and its members are shown in the Table.

The Table shows that it is difficult to distinguish between the species. Williams and Hammond ${ }^{10}$ identified three groups of capnocytophaga using DNA-DNA hybridisation techniques, from which they concluded that there were three distinct species. They found the intergroup divergence greater than expected from the known phenotypic characteristics. Collins et al, ${ }^{13}$ however, who examined 13 strains, of which only two or possibly three were used in the DNA-DNA homology studies, ${ }^{10}$ considered it unlikely that such high interspecies DNA-DNA homology values would occur while the DNA base composition has such a wide range-for example, $C$ gingivalis, DNA-DNA homology range $100-72 \%,{ }^{10}$ has a mol \% G+C range of $35-41$.

It is interesting that in cases of systemic disease, if the isolate has been speciated it is usually reported as $C$ ochracea. Because of the difficulties outlined above in speciation of Capnocytophaga spp it would be unwise to suggest that $C$ ochracea is more pathogenic than the other species. As further data accumulate on capnocytophaga infections, however, the pathogenicity of the various species should become clearer.

Studies of the antigenic components of Capnocytophaga show that two antigens exist, both probably located in the cell wall. ${ }^{14}$ The group specific antigen $(G)$ was found in all 26 strains tested and consists mainly of protein. Three strains also possessed a type specific antigen $(T)$, which was predominantly carbohydrate..$^{14}$ In another study of the cell surface components of capnocytophaga all the currently recognised species contained a sulphonolipid unique to the procaryotes, which has been assigned the name capnine. It was suggested that capnine might in some way be related to the gliding motility exhibited by these organisms. ${ }^{15}$

Capnocytophaga species have also been reported to possess a characteristic cellular fatty acid profile, which enables them to be distinguished from other gliding bacteria but not from one another..$^{13} 1617$

\section{ISOLATION}

Several workers have emphasised that Capnocytophaga spp are not isolated from clinical specimens by standard laboratory techniques. ${ }^{3418}$ As the name of the organism suggests, it requires an atmosphere with an increased carbon dioxide content for growth. Also, some strains may require strict anaerobic conditions for primary isolation. In a study of the effect of different atmospheres of incubation on the growth of capnocytophaga, atmospheres of pure hydrogen or pure nitrogen failed to support growth; however, concentrations of $\mathrm{CO}_{2}$ between $5 \%$ and $100 \%$ all supported good growth. ${ }^{2}$ Anaerobic gas mixtures (nitrogen/hydrogen/carbon dioxide) have also been used for isolation; they are adequate provided that at least $5 \% \mathrm{CO}_{2}$ is present. ${ }^{413}$ The absolute growth requirement for $\mathrm{CO}_{2}$ seen in C ochracea is due to the organism's total dependence of fixing $\mathrm{CO}_{2}$ to incorporate into its intermediary carbohydrate metabolism. The only enzyme responsible for $\mathrm{CO}_{2}$ fixation in Cochracea is phosphoenolpyruvate carboxykinase, an enzyme found in other bacteria that require $\mathrm{CO}_{2}$ for growth. ${ }^{19}$ Capnocytophaga spp will grow on most enriched laboratory media, as reported in this paper. Trypticase soy agar with $5 \%$ sheep blood has been reported to give maximal growth.' A selective medium for the recovery of Capnocytophaga spp has also been reported which consists of trypticase soy agar containing $5 \%$ sheep blood, $50 \mu \mathrm{g} / \mathrm{ml}$ bacitracin, and $100 \mu \mathrm{g} / \mathrm{ml}$ polymyxin B. ${ }^{18}$ Although this medium is reported to be useful for distinguishing capnocytophaga in mixed populations of bacteria, clinical microbiology laboratories will find that prolonged incubation of existing media such as bacitracin chocolate agar will give adequate results.

\section{OCCURRENCE AND PATHOGENICITY}

The principal natural habitat in man of capnocytophaga appears to be the mouth. $C$ ochracea occurs in the subgingival sulcus of healthy adults. ${ }^{20}$ Although found in the healthy gingiva, much larger number of capnocytophaga may be isolated from cases of periodontitis. ${ }^{21}$ It is not the intention of this review to discuss the role of capnocytophaga in periodontitis in great depth; this has been discussed in detail elsewhere. ${ }^{22} 7$

It is the recently recognised ability of capnocytophaga to produce systemic disease in immunocompromised patients that is of greatest 
interest to clinical microbiologists. Most cases have been seen in patients with haematological malignancy and profound neutropenia, often with oral mucosal ulceration, which is presumed to be the portal of entry of the organisms to the bloodstream. A number of single case histories of septicaemia in immunocompromised patients ${ }^{45-26}$ and a series of six patients ${ }^{3}$ have been published, all from the USA. Apart from three patients ${ }^{543}$ all patients survived after appropriate chemotherapy and all were diagnosed as having had capnocytophaga infection by prolonged incubation of blood cultures and subcultures. It is interesting that in the report of six patients, ${ }^{3}$ none of the patients was receiving an antibiotic effective against capnocytophaga when the episode of sepsis occurred.

Apart from the mouths and the blood of septicaemic immunocompromised patients, capnocytophaga has also, though less often, been isolated from sputum and upper respiratory tract specimens such as throat swabs and transtracheal aspirates. ${ }^{8}$ This confirms the predominantly oral habitat of the organism in man, although it has also rarely been found in the female genital tract. ${ }^{7}$ Disease caused by capnocytophaga in the respiratory tract is rare, although pneumonia ${ }^{5}$ and an empyema in a patient with carcinoma of the bronchus ( Dr WMN Nicholls, personal communication) have been reported. The second patient was not neutropenic, although aspiration of saliva was thought to be the source of infection in both cases.

Reports of the occurrence of disease caused by $C$ ochracea in non-immunocompromised patients are rare. Cochracea was reported as the cause of a cervical abscess in a normal adolescent boy in Switzerland. ${ }^{27} \mathrm{~A}$ single case of endocarditis caused by $C$ ochracea has also been reported in a nonimmunocompromised patient, which was successfully treated with amoxycillin and metronidazole..$^{28}$ Recently, a case of septic arthritis of the knee caused by $C$ ochracea was described in a 21 month old child. ${ }^{29}$ Together with our own case, these are the only reported systemic infections due to this organism in non-neutropenic patients.

The interaction between capnocytophaga and the host defences appears to be complex. It produces a dialysable substance which impairs neutrophil chemotaxis, The morphological changes induced in neutrophils by this substance in vitro were also seen in two patients infected with capnocytophaga. ${ }^{23}$ In addition, $C$ ochracea is able to degrade $\operatorname{IgA} 1$ (but not IgA2) and IgG, which would enable the organism to induce local paralysis of the host defences. ${ }^{30}$ Another member of the group, $C$ sputigena produces a fibroblast proliferation inhibitory factor ${ }^{30}$ and an endotoxin. ${ }^{32}$ The precise roles of these vari- ous virulence factors in the evolution of disease caused by capnocytophaga have not yet been evaluated.

\section{ANTIMICROBIAL SUSCEPTIBILITY}

Some antimicrobial regimens used in the blind treatment of suspected episodes of infection in immunocompromised patients do not provide adequate activity against capnocytophaga. An in vitro study of antimicrobial susceptibility suggested that clindamycin, penicillin, erythromycin, ampicillin, or carbenicillin are the antibiotics of choice for treatment of infection caused by capnocytophaga. The activities of most first and second generation cephalosporins (except cefoxitin), vancomycin, and oxacillin were poor and unpredictable; the isolates were also resistant to aminoglycosides. Metronidazole, chloramphenicol, and tetracycline were also moderately active against the strains tested. ${ }^{33} \mathrm{~A}$ further in vitro study of 27 isolates confirmed the findings of Forlenza et al. ${ }^{33}$ Sutter et al ${ }^{34}$ used latamoxef as the only representative of the expanded spectrum $\beta$-lactams and found it to be active $\left(\mathrm{MIC}_{50} 1 \mathrm{mg} / \mathrm{l}\right)$. The isolate from case 2 reported here was sensitive to ceftazidime and the patient responded to treatment with that agent. The activity of many third generation cephalosporins was not reported in these studies ${ }^{33}{ }^{34}$; it would be of interest as they are increasingly used to treat immunocompromised patients.

Plasmid mediated drug resistance has been reported in $C$ ochracea. Two plasmids were identified; the larger pGD10 (70 megadaltons) specified resistance to chloramphenicol, tetracycline, kanamycin, and streptomycin; the smaller (25 megadaltons) was a cryptic plasmid with no identifiable phenotypic markers. ${ }^{35}$ Further studies of pDG10 have shown that it belongs to the wide host range incompatibility group FII and has a similar host range to other Inc FII plasmids. Digestion of plasmid DNA from pDG10 with restriction endonucleases showed that it was closely related to other widely distributed $R$ factors such as $R 100, R 1$, and $\mathrm{R} 6 .^{36}$

In conclusion, members of the genus capnocytophaga are recently recognised pathogens causing oral ulceration in neutropenic patients, which may progress to life threatening septicaemia. They may also cause systemic disease in immunologically normal hosts. The clinical microbiologist should be alerted to the possible presence of this organism if a specimen of pus with an apparently negative Gram film from a site related to the respiratory tract fails to grow a recognisable bacterial pathogen after $48 \mathrm{~h}$ incubation. 
We thank Mr RN Baird and Dr JS Lilleyman for permission to report on their patients.

The two strains described in this paper have been deposited in the National Type Culture Collection and are designated as follows: A319/83: NCTC 11654, A349/83: NCTC 11655.

\section{References}

' Newman MG, Weuner MS, Angel I, Grinenko V, Karge HJ. Predominant cultivable microbiota of the gingival crevice in "supernormal" patients. [Abstract. 278]. J Dent Res 1977;56:B121.

${ }^{2}$ Socransky SS, Holt SC, Leadbetter ER, Tanner ACR, Savitt E, Hammond BF. Capnocytophaga: New genus of Gramnegative gliding bacteria. III. Physiological characterisation. Arch Microbiol 1979;122:29-33.

${ }^{3}$ Forlenza SW, Newman MG, Lipsey AI, Siegel SE, Blachman UL. Capnocytophaga sepsis: A newly recognised clinical entity in granulocytopenic patients. Lancet 1980;i:567-8.

4 Gilligan PH, McCarthy LR, Bissett BK. Capnocytophaga ochracea septicaemia. J Clin Microbiol 1981;13:643-5.

s Shlaes DM, Dul MJ, Lerner PI. Capnocytophaga bacteraemia in the compromised host. Am J Clin Pathol 1982;77:359-61.

- Reichenbach H. Taxonomy of the gliding bacteria. Ann Rev Microbiol 1981;35:339-64.

' Forlenza S, Newman MG. Unusual microorganisms-Gramnegative fastidious species. In: Bottone EJ, ed. Capnocytophaga. New York: Marcel Dekker Inc, 1983:45-6.

${ }^{*}$ Newman MG, Sutter VL, Pickett MJ, et al. Detection, identification, and comparison of Capnocytophaga, Bacteroides ochraceus, and DF-1. J Clin Microbiol 1979;10:55762.

" Williams BL, Hollis D, Holdeman LV. Synonomy of strains of Center for Disease Control group DF-1 with Species of Capnocytophaga. J Clin Microbiol 1979;10:550-6.

${ }^{10}$ Williams BL, Hammond BF. Capnocytophaga: New genus of Gram-negative bacteria. IV. DNA base composition and sequence homology. Arch Microbiol 1979;122:35-9.

"Leadbetter ER, Holt SC, Socransky SS. Capnocytophaga: new genus of Gram-negative gliding bacteria. I. General characteristics, taxonomic considerations and significance. Arch Microbiol 1979;122:9-16.

12 Cowan ST. Cowan and Steel's manual for the identification medical bacteria. 2nd ed. London: Cambridge University Press, 1974.

${ }^{13}$ Collins MD, Shah HN, McKee AS, Kroppenstedt RM. Chemotaxonomy of the genus Capnocytophaga (Leadbetter. Holt \& Socransky). J Appl Bacteriol 1982;52:409-15.

${ }^{14}$ Stevens $\mathrm{RH}, \mathrm{Hammond} \mathrm{BF}$, Lai $\mathrm{CH}$. Group and type antigens of Capnocytophaga. Infect Immun 1979;23:532-9.

is Godchaux W, Leadbetter ER. Capnocytophaga spp contain sulfonolipids that are novel in procaryotes. $J$ Bacteriol 1980; 144:592-602.

${ }^{16}$ Holt SC, Forcier G, Takacs BJ. Fatty acid composition of gliding bacteria: Oral isolates of Capnocytophaga compared with Sporocytophaga. Infect Immun 1979;26: 298-304.

17 Dees SB, Karr DE, Hollis D, Moss CW. Cellular fatty acids of
Capnocytophaga species. J Clin Microbiol 1982;16:779-83.

${ }^{1 *}$ Mashimo PA, Yamamoto Y, Nakamura M, Slots J. Selective recovery of oral Capnocytophaga spp with sheep blood agar containing bacitracin and polymyxin B. J Clin Microbiol 1983; 17: 187-91.

${ }^{19}$ Kapke PA, Brown AT, Lillich TT. Carbon dioxide metabolism by Capnocytophaga ochracea: Identification, characterization, and regulation of a phosphoenolpyruvate carboxykinase. Infect Immun 1980;27:756-66.

${ }^{20}$ Moore WEC, Holdeman LV, Cato EP, Smibert RM, Burmeister JA, Ranney RR. Bacteriology of moderate (chronic) periodontitis in mature adult humans. Infect Immun 1983;42:510-5.

${ }^{21}$ Newman MG, Socransky SS, Savitt ED, Propas D, Crawford A Studies of the microbiology of periodontosis. J Periodont 1976:47:373-9.

${ }^{22}$ Socransky SS. Microbiology of periodontal disease-present status and future considerations. J Periodont 1977;48:497504

${ }^{23}$ Shurin SB, Socransky SS, Sweeney E, Stossel TP. A neutrophil disorder induced by Capnocytophaga, a dental microorganism. N Engl J Med 1979;301:849-54.

${ }^{24}$ Appelbaum PC, Ballard JO, Eyster ME. Septicaemia due to Capnocytophaga (Bacteroides ochraeus) in Hodgkin's disease. Ann Intern Med 1979;90:716-7.

${ }^{25}$ Gandola C, Butler T, Badger S, Cheng E, Beard S. Septicaemia caused by Capnocytophagas in a granulocytopenic patient witi glossitis. Arch Intern Med 1980; 140:851-2.

${ }^{26}$ Haulk AA, Sugar AM, Rushing JL. Capnocytophaga septicaemia. $N$ Engl J Med 1980; 302:922.

27 Seger R, Kloeti J, von Gravenitz A, Wust J, Briner J, Willi U, Siergrist H. Cervical abscess due to Capnocytophaga ochracea. Paediatric Infectious Disease 1982;1:170-2.

${ }^{2 \times}$ Coignard S, Malbrunot C, Bor Y, Perrot S, Rouveix H, Dechy H, 8 Dorra M. Endocardite a Capnocytophaga ochracea. Nouv Presse Med 1982;11:1338.

${ }^{24}$ Winn RE, Chase WF, Lauderdale PW, McClesky FK. Septic arthritis involving Capnocytophaga ochracea. J Clin Microbiol 1984; 19:538-40.

${ }^{30}$ Kilian M. Degradation of immunoglobulins A1, A2 and G by suspected principal periodontal pathogens. Infect Immun 1981;34:757-65.

${ }^{31}$ Stevens RH. Sela MN. Shapira J, Hammond BF. Detection of a fibroblast proliferation inhibitory factor from Capnocytophaga sputigena. Infect Immun 1980;27:271-5.

${ }^{32}$ Stevens RH, Sela MN, McArthur WP, Nowotny A, Hammond BF. Biological and chemical characterization of endotoxin from Capnocytophaga sputigena. Infect Immun 1980;27:246-54.

${ }^{33}$ Forlenza SW, Newman MG, Horikoshi AL, Blachman U. Antimicrobial Susceptibility of Capnocytophaga. Antimicrob Agents Chemother 1981;19:144-6.

${ }^{34}$ Sutter VL, Pyeatt D, Kwok YY. In vitro susceptibility of Capnocytophaga stains to 18 antimicrobial agents. Antimicrob Agents Chemother 1981;20:270-1.

${ }^{35}$ Guiney DG, Davis CE. Identification of a conjugative $R$ plasmid in Bacteroides ochracea capable of transfer to Escherichia coli. Nature 1978:274:181-2.

${ }^{36}$ Guiney DG, Davis CE. Incompatibility and host range of pGD10 from Capnocytophaga ochraceus, formerly Bacteriodes ochraceus. Plasmid 1982;7:196-8. 